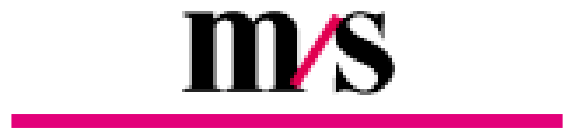

médecine/sciences $1995 ; 11$ : 559-62

\title{
DES SOURIS ET DES HOMMES : L'HYPERTENSION À L'ĖRE DE LA GÉNÉTIQUE MOLÉCULAIRE
}

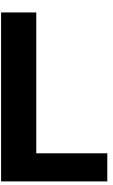

a recherche concernant l'hypertension artérielle semble avoir finalement rattrapé les approches révolutionnaires de la médecine moléculaire. En effet, durant les années 1990, les chercheurs ont pu largement dépasser l'étape corrélative pour identifier, grâce à plusieurs approches de génétique moléculaire, des gènes directement impliqués dans le développement de l'hypertension artérielle.

Dans la majorité des pays industrialisés, l'hypertension essentielle - une augmentation de la pression artérielle de cause inconnue - est un problème majeur qui affecte près de $25 \%$ de la population adulte et qui constitue l'un des facteurs de risque majeurs des maladies cardio- et cérébrovasculaires. S'il existe aujourd'hui différentes classes de médicaments palliatifs qui permettent de contrôler la pression artérielle, leur efficacité est variable et, dans tous les cas, il s'agit de traitements à vie ayant des effets secondaires et des inconvénients typiques de toute thérapie de longue durée. Par ailleurs, les mesures préventives, telle une alimentation faible en sel, se heurtent à l'hétérogénéité de la maladie ; en effet, l'hypertension chez certains patients est sensible au sel alors que, chez d'autres, l'augmentation de la pression artérielle est totalement indépendante de l'apport sodé. En outre, même si l'aspect héréditaire de l'hypertension est reconnu, le mode de transmission génétique est loin d'être établi (voir l'article de A. Bonnardeaux, p. 575 de ce numéro). En effet, l'hypertension est une maladie complexe avec des composantes multigéniques et liées à l'environnement, ce qui rend l'analyse génétique beaucoup plus compliquée. Ainsi, plusieurs gènes qui contribueraient chacun modestement à l'augmentation de la pression seraient impliqués et c'est seulement la combinaison de deux gènes ou plus, ou d'un gène combiné à des facteurs d'environnement, tels le stress ou une alimentation riche en sel, qui seraient responsables de l'état hypertendu. D'autre part, il n'est pas exclu que le phénotype clinique comprenne lui-même plusieurs sous-groupes de maladies aux étiologies différentes mais qui aboutissent toutes aux mêmes manifestations cliniques. Autrement dit, s'agit-il vraiment de l'hypertension ou doit-on plutôt envisager des hypertensions? Historiquement, la recherche sur l'hypertension a pu bénéficier de la disponibilité de plusieurs modèles d'hypertension chez le rat incluant des modèles génétiques d'hypertension indépendante du sel (les rats spontanément hypertendus) et induite par le sel (les rats Dahl). Outre leur utilité évidente pour analyser l'expression de gènes « candidats» et pour tester des pro- 
duits antihypertenseurs (voir par exemple l'article de E. Schiffrin, p. 599 de ce numéro), ces animaux sont des modèles expérimentaux précieux pour élucider la pathogénie de l'hypertension et, en particulier, pour des études de liaison génétique. Telle qu'elle est discutée dans l'article de A. Bonnardeaux, l'analyse de gènes candidats ou l'étude systématique du génome dans des croisements génétiques contrôlés chez ces modèles a permis récemment d'identifier plusieurs loci associés à l'hypertension dont certains ont déjà été confirmés chez l'homme. La découverte de gènes liés à la pression artérielle est évidemment un pas de géant vers la compréhension de la pathogénie de l'hypertension. Il ne faut cependant pas oublier que les études de liaison génétique identifient des loci qui ségrègent avec le phénotype étudié mais ne prouvent pas une relation causale. Autrement dit, il est possible que le gène pertinent soit lui-même lié au locus identifié qui sert alors simplement de marqueur. C'est là que la production d'animaux transgéniques chez lesquels un gène spécifique a été altéré offre un moyen des plus puissants pour déterminer la relation causale entre un gène et un phénotype (voir par exemple la mini-synthèse de R.L. Joshi et J. Jami, p. 620 de ce numéro). C'est ainsi que la production d'animaux transgéniques - chez lesquels des gènes possiblement liés à l'hypertension ont été, soit surexprimés, soit inactivés - a révélé, confirmé ou écarté l'implication de certains systèmes et/ou molécules dans le développement de l'hypertension. En outre, cette approche a engendré une gamme impressionnante de nouveaux modèles expérimentaux qui pourraient représenter des sous-types d'hypertension essentielle chez l'homme.

\section{Le système rénine/angiotensine : le crime organisé}

Le maintien d'une pression normale est assuré par l'interaction de plusieurs systèmes qui contrôlent le débit cardiaque, les résistances périphériques, le volume sanguin ainsi que la fonction rénale et l'équilibre sodé. Ces systèmes subissent euxmêmes des influences hormonales et nerveuses. L'une des hormones qui joue un rôle majeur dans le contrôle de la pression sanguine et de l'équilibre des électrolytes est l'angiotensine II (AII). AII est le produit mûri de l'angiotensinogène (Ang) qui est sécrété essentiellement par le foie. La rénine, enzyme sécrétée par le rein, coupe l'Ang pour engendrer l'angiotensine I qui sera, à son tour, clivée par une autre enzyme, l'enzyme de conversion de l'angiotensine (ACE), pour produire l'hormone active AII. AII est une hormone hypertensive qui agit sur plusieurs organes via deux types de récepteurs membranaires, AT1 et AT2, pour stimuler, entre autres, la contraction $\mathrm{du}$ muscle lisse vasculaire et la sécrétion de l'aldostérone. Outre la production périphérique de l'AII, il existe des systèmes rénine-angiotensine locaux qui pourraient agir de manière autocrine. De tels systèmes par exemple sont trouvés dans la glande surrénale et dans le cœur. Les niveaux de rénine et AII sont normaux ou réduits chez les patients manifestant une pression artérielle élevée. Cependant, l'implication du système rénine-angiotensine dans l'hypertension a été mise en évidence grâce à plusieurs approches moléculaires dont les études génétiques de liaison dans des modèles animaux et des populations humaines et surtout par la production de modèles transgéniques de surexpression ou d'inactivation de chacun des gènes codant pour les différents éléments du système. Ainsi, en 1990 Mullins et al. [1] ont créé des rats transgéniques qui surexpriment le gène rénine Ren-2 de souris dans les glandes surrénales $\left(\mathrm{m} / \mathrm{s} n^{\circ} 8\right.$, vol. 6, p. 826); ces rats développent une hypertension sévère avec des manifestations semblables à l'hypertension dite "rénine-faible » chez l'homme et représenteraient un sous-type d'hypertension causée par un dérèglement fonctionnel de la glande surrénale. La même année, Ohkubo et al. [2] créaient un autre modèle transgénique d'hypertension en surexprimant chez des souris transgéniques les gènes rénine et angiotensinogène de rat $\left(\mathrm{m} / \mathrm{s} n^{\circ} 8\right.$, vol. 6, p. 826). D’autres études transgéniques ont par la suite confirmé ces résultats (par exemple [3, 4]). Ces travaux ont non seulement affirmé le rôle important du système rénine-angiotensine mais ont, de plus, annoncé une nouvelle ère dans la recherche en hypertension.

Si les études de surexpression ont conforté un rôle pathologique du système rénine-angiotensine, la confirmation d'un rôle physiologique a été obtenue grâce aux techniques d'inactivation génique (knock-out). En effet, l'inactivation du gène $A n g$ chez la souris a donné naissance à des lignées de souris hypotendues $[5,6]$ indiquant que l'AII est indispensable au maintien de la pression artérielle. Le même phénotype hypotendu fut observé chez les souris ne synthétisant plus le récepteur AII de type 1A [7]. Ce dernier résultat n'était pas évident a priori, considérant qu'un autre récepteur existe pour AII (récepteur de type 2 ou AT2); le rôle du récepteur AT2 est, en effet, resté plutôt énigmatique jusqu'au développement récent de souris transgéniques chez lesquelles le gène AT2 était inactivé $\left(\mathrm{m} / \mathrm{s} n^{\circ} 1\right.$, vol. 12 , p. 122) $[8,9]$. Dans ce cas, les souris étaient hypertendues avec une sensibilité accrue à l'AII indiquant que le récepteur AT2 sert à contrebalancer l'effet hypertenseur du récepteur AT1A. Ces animaux présentaient, en outre, des changements significatifs de comportement, impliquant le récepteur AT2 dans la régulation des systèmes cardio-vasculaire et nerveux. Enfin, le gène codant pour l'ACE a été inactivé [10] et a révélé pour la première fois une base moléculaire pour le dimorphisme sexuel dans l'hypertension $\left(\mathrm{m} / \mathrm{s} n^{\circ} 10\right.$, vol. 11 , p. 1496). Ainsi, bien que l'activité plasmatique de l'ACE était réduite de façon semblable chez des hétérozygotes mâles et femelles, seuls les mâles avaient une réduction importante de la pression artérielle. Les études de liaison dans des modèles animaux et des études de cas chez l'homme avaient déjà suggéré un lien entre le locus ACE et l'hypertension (voir l'article d'A. Bonnardeaux, p. 575 de ce numéro). L'inactivation du gène $A C E$ dans un contexte génétique très bien défini confirme que 
les mutations identifiées chez des patients hypertendus sont bel et bien impliquées dans le développement de l'hypertension humaine.

\section{Les peptides natriurétiques ou le dilemme salé}

Les diverses actions hypertensives de AII sont contrecarrées par deux hormones hypotensives produites essentiellement par le cour, le peptide natriurétique auriculaire (ANP) et le peptide natriurétique de type $B$ (BNP). En dépit des effets hypotenseurs de ces deux hormones, leur rôle dans l'hypertension est resté longtemps controversé, la raison principale étant le manque de corrélation entre les niveaux plasmatiques de l'ANP et la pression artérielle chez l'homme. Cependant, les concentrations de l'ANP étaient significativement plus élevées chez les patients hypertendus à l'apport sodé non contrôlé comparés à des normotendus, suggérant que l'ANP serait plutôt lié à l'hypertension sensible au sel. Cette hypothèse a été confirmée par la production de souris transgéniques au gène $A N P$ inactivé [11]. En effet, les souris hétérozygotes n'avaient une pression élevée qu'en réponse à l'augmentation du sel dans leur alimentation, indiquant qu'une diminution de l'ANP serait suffisante pour causer une hypertension sensible au sel. Il reste cependant à chercher si un dérèglement de l'ANP existe dans une sous-population de patients dont l'hypertension est sensible au sel. Une telle sous-population pourrait correspondre au groupe de patients ayant montré une réponse favorable aux inhibiteurs des endopeptidases neutres, des antihypertenseurs qui agissent essentiellement en augmentant le niveau circulant de l'ANP [12]. Que l'inactivation du gène de l'ANP produise un phénotype d'hypertension était loin d'être garanti. En effet, on aurait pu imaginer que l'autre peptide de la famille, le BNP qui possède les mêmes activités biologiques que l'ANP et qui se lie au même récepteur membranaire, pût compenser l'absence de l'ANP. Ainsi, le phénotype des souris $A N P$-/-indique un rôle spécialisé pour chacune de ces hormones. Le phénotype des souris chez lesquelles le gène du récepteur de type A (GC-A) des peptides natriurétiques a été inactivé, doit être analysé dans ce contexte. Les souris GCA-/sont hypertendues indépendamment du sel, suggérant qu'au moins un des ligands de ce récepteur - vraisemblablement BNP - est essentiel au maintien de la pression artérielle normale [13]. On attend donc avec impatience les résultats de l'inactivation du gène $B N P$. Néanmoins, le phénotype GCA-/- ressemble à l'hypertension de près de $50 \%$ des patients souffrant d'une hypertension insensible au sel avec production normale d'aldostérone suggérant que, là encore, des composantes du système des peptides natriurétiques pourraient être directement liées à l'affection.

\section{L'endothéline : coupable par association ?}

Les propriétés vasoconstrictrices de l'endothéline 1 (ET-1), un peptide initialement isolé des cellules endothéliales vasculaires, ont suggéré qu'une dérégulation du système endothéline pouvait être liée à l'hypertension même en l'absence d'une corrélation entre les niveaux plasmatiques d'ET-1 et la pression sanguine (voir l'article de E. Schiffrin p. 599 de ce numéro). ET-1 appartient à une famille de peptides apparentés qui agissent via deux types de récepteurs membranaires, les récepteurs ETA et ETB (voir l'article de P. d'Orléans-Juste p. 563 de ce numéro). L'inactivation des gènes de ET-1, ET3 et du récepteur ETA chez la souris [14-16] a bouleversé l'hypothèse selon laquelle l'endothéline aurait été essentiellement un vasoconstricteur qui contribue au maintien de la pression artérielle. De fait, cette approche qui fait abstraction des preuves indirectes, a révélé un rôle tout à fait inattendu des endothélines dans le développement embryonnaire et, en particulier, dans les tissus dérivés de la crête neurale $\left(\mathrm{m} / \mathrm{s} \mathrm{n}^{\circ} \mathrm{6}\right.$ 7, vol. 10, p. 740). Ainsi, les souris ET3-/- ou ET-B -/- ont le même phénotype de pigmentation et de mégacôlon typiques de la maladie d'Hirschsprung et des mutations spontanées du gène $E T-B$ sont associées à cette maladie $\left(m / s n^{\circ} 8\right.$, vol. $11, p$. 1172). De tels résultats inattendus présentent évidemment un défi aux nombreuses études corrélatives mais illustrent aussi la puissance et l'utilité des approches de génétique moléculaire.

\section{Des gènes et encore des gènes d'hypertension}

Étant donné la multitude de systèmes impliqués dans le contrôle de la pression artérielle normale, il est tout à fait possible qu'outre les systèmes décrits ci-dessus, les techniques d'inactivation génique identifient encore d'autres gènes impliqués dans l'hypertension. Déjà, le rôle du monoxyde d'azote (NO) dans le maintien de la pression artérielle a été mis en évidence par inactivation de l'isoforme endothéliale de la NO synthase $\left(\mathrm{m} / \mathrm{s} n^{\circ} 12\right.$, vol. $\left.11, p .1743\right)$ [17]. Il serait aussi intéressant de croiser les diverses lignées de souris hypertendues pour évaluer les contributions coopératives des différents systèmes et essayer de créer de véritables modèles polygéniques d'hypertension. Enfin, dans les pays industrialisés, le stress est probablement un facteur permissif au développement de certaines hypertensions qu'il faudra essayer de tester chez les souris génétiquement altérées

\section{TIRÉS À PART}

M. Nemer

\section{RÉFÉRENCES}

1. Mullins JJ, Peters J, Ganten D. Fulminant hypertension in transgenic rats harbouring the mouse Ren-2 gene. Nature $1990 ; 344$ : 541-4.

2. Ohkubo H, Kawakami H, Kakehi Y, Takumi T, Arai H, Yokota Y, Iwai M, Tanabe Y, Masu M, Hata J, et al. Generation of transgenic mice with elevated blood pressure by introduction of the rat renin and angiotensinogen genes. Proc Natl Acad Sci USA 1990 ; 87: 5153-7. 


\section{RÉFÉRENCES}

3. Yang G, Merrill DC, Thompson MW, Robillard JE, Sigmund CD. Functional expression of the human angiotensinogen gene in transgenic mice. J Biol Chem 1994; 269 : 32497-502.

4. Kimura S, Mullins JJ, Bunnemann B, Metzger R, Hilgenfeldt U, Zimmermann F, Jacob H, Fuxe K, Ganten D, Kaling M. High blood pressure in transgenic mice carrying the rat angiotensinogen gene. $E M B O \mathrm{~J}$ $1992 ; 11: 821-7$.

5. Tanimoto K, Sugiyama F, Goto Y, Ishida J, Takimoto E, Yagami K, Fukamizu A, Murakami K. Angiotensinogen-deficient mice with hypotension. J Biol Chem 1994; 269 : 31334-7.

6. Kim HS, Krege JH, Kluckman KD, Hagaman JR, Hodgin JB, Best CF, Jennette JC, Coffman TM, Maeda N, Smithies O. Genetic control of blood pressure and the angiotensinogen locus. Proc Natl Acad Sci USA 1995 ; 92 : 2735-9.

7. Ito M, Oliverio MI, Mannon PJ, Best CF, Maeda N, Smithies O, Coffman TM. Regulation of blood pressure by the type 1A angiotensin II receptor gene. Proc Natl Acad Sci USA 1995 ; 92 : 3521-5.
8. Ichiki T, Labosky PA, Shiota C, Okuyama S, Imagawa Y, Fogo A, Niimura F, Ichikawa I, Hogan BL, Inagami T. Effects on blood pressure and exploratory behaviour of mice lacking angiotensin II type-2 receptor. Nature 1995 ; 377 : 748-50.

9. Hein L, Barsh GS, Pratt RE, Dzau VJ, Kobilka BK. Behavioural and cardiovascular effects of disrupting the angiotensin II type2 receptor in mice. Nature 1995 ; 377 : 744-7.

10. Krege JH, John SW, Langenbach LL, Hodgin JB, Hagaman JR, Bachman ES, Jennette JC, O'Brien DA, Smithies O. Malefemale differences in fertility and blood pressure in ACE-deficient mice. Nature $1995 ; 375$ : 146-8.

11. John SW, Krege JH, Oliver PM, Hagaman JR, Hodgin JB, Pang SC, Flynn TG, Smithies O. Genetic decreases in atrial natriuretic peptide and salt-sensitive hypertension. Science 1995 ; 267 : 679-81.

12. Ogihara T, Rakugi H, Masuo K, Yu H, Nagano M, Mikami H. Antihypertensive effects of the neutral endopeptidase inhibitor SCH 42495 in essential hypertension. Am J Hypertens 1994 ; 7 : 943-7.

13. Lopez MJ, Wong SK, Kishimoto I, Dubois S, Mach V, Friesen J, Garbers DL, Beuve A.
Salt-resistant hypertension in mice lacking the guanylyl cyclase-A receptor for atrial natriuretic peptide. Nature 1995 ; 378 : 65-8.

14. Kurihara Y, Kurihara H, Suzuki H, Kodama T, Maemura K, Nagai R, Oda H, Kuwaki T, Cao WH, Kamada N, Jishage K, Ouchi Y, Azuma S, Toyoda Y, Ishikawa T, Kumada M, Yazaki Y. Elevated blood pressure and craniofacial abnormalities in mice deficient in endothelin-1. Nature $1994 ; 368$ : 703-10.

15. Baynash AG, Hosoda K, Giaid A, Richardson JA, Emoto N, Hammer RE, Yanagisawa M. Interaction of endothelin-3 with endothelin-B receptor is essential for development of epidermal melanocytes and enteric neurons. Cell $1994 ; 79$ : 1277-85.

16. Hosoda K, Hammer RE, Richardson JA, Baynash AG, Cheung JC, Giaid A, Yanagisawa M. Targeted and natural (piebald-lethal) mutations of endothelin-B receptor gene produce megacolon associated with spotted coat color in mice. Cell 1994 ; 79 : 1267-76.

17. Huang PL, Huang Z, Mashimo H, Bloch KD, Moskowitz MA, Bevan JA, Fishman MC. Hypertension in mice lacking the gene for endothelial nitric oxide synthase. Nature 1995 ; 377 : 239-42. 